\title{
A fully elastic model for studying submerged circular cylindrical shells subjected to a weak shock wave
}

\author{
Cédric Leblond ${ }^{1, a}$, Serguei Iakovlev ${ }^{2}$ and Jean-Francois Sigrist ${ }^{3}$ \\ 1 LEPTIAB, Université de La Rochelle, Avenue Michel Crépeau, 17042 La Rochelle Cedex 1, France \\ 2 Dept of Engineering Mathematics and Internetworking, Dalhousie University, Halifax, Canada B3J 2X4 \\ 3 Service Technique et Scientifique, DCNS Propulsion Indret, BP 30, 44620 La Montagne, France
}

Received 20 April 2009

\begin{abstract}
The transient dynamics of evacuated and fluid-filled circular elastic shells, submerged in an infinite fluid medium and subjected to an external weak shock wave, is considered in this paper. This circular shell/acoustic medium interaction problem has already been tackled with simplified thin shell models, based on the Love-Kirchhoff hypotheses for the structural dynamics. In this case, the resulting radiated pressure field displays some discrepancies related to the $A_{0} / S_{0}$ waves when compared to the experimental data available in the literature for the evacuated case. These drawbacks are overcome here by the use of an isotropic elastic model for the structural dynamics and an inviscid acoustic flow for the fluid dynamics, in a two-dimensional framework. The approach is based on the methods of Laplace transform in time, Fourier series expansions and separation of variables in space. For the fluid-filled case, the transient thick shell-weak shock wave interaction problem is explored and the radiated acoustic field described.
\end{abstract}

Key words: Circular shell / elasticity / acoustic / wave propagation / underwater explosion / fluidstructure interaction

\begin{abstract}
Résumé - Un modèle élasto-dynamique pour l'étude de la dynamique de coques immergées assujetties à une onde de choc de faible intensité. La dynamique transitoire de coques circulaires vides ou contenant un fluide, immergées dans un milieu fluide infini et assujetties à une onde de choc de faible intensité, est considérée ici. Ce problème d'interaction a déjà été traité dans la littérature à l'aide de modèles de coques minces basés sur les hypothèses de Kirchhoff-Love. Dans ce cas, le champ de pression rayonné par la coque présente des différences significatives par rapport aux données expérimentales disponibles dans la littérature pour une coque vide. Ces lacunes sont éliminées ici par l'utilisation d'un modèle élastique, résolu semi-analytiquement. L'approche est basée sur les méthodes de transformations de Laplace en temps, expansions en série de Fourier et séparations des variables en espace. Dans le cas d'une coque remplie de fluide, le problème d'interaction transitoire est exploré et le champ rayonné est décrit.
\end{abstract}

Mots clés : Coque circulaire / élasticité / acoustique / propagation d'ondes / explosion sous-marine / interaction fluide-structure

\section{Introduction}

Underwater shock analysis is of paramount importance in various industries for which the need to design structures towards the effects of underwater explosion is required, for instance in offshore engineering and of course in naval shipbuilding. As far as this latter is concerned, the underwater shock analysis is one of the main requirements in design or even pre-design studies of surface ships and submarines: it necessitates both

\footnotetext{
${ }^{a}$ Corresponding author: cedric.leblond@univ-lr.fr
}

global (explosion/ship-hull/free-surface interactions) and local (effects of the hydrodynamic shock at various location within the ship) analyses, which are to be conducted throughout the design process of the ship and its equipments.

Underwater explosions [1] and their effects on immersed structures involve rather complex multi-physic (from chemical modelling of the explosive to coupled fluid-structure interactions between the weak shock wave and immersed structures, ship hull in particular) and multi-scale (global and local fluid-structure interactions) 


\section{Nomenclature}

\begin{tabular}{|c|c|}
\hline $\mathbf{a}_{\mathbf{n}}(s)=\left\{A_{n}(s), B_{n}(s), C_{n}(s), D_{n}(s)\right\}^{T}$ & unknown column vector \\
\hline$c_{l}$ & longitudinal wave velocity in the shell \\
\hline$c_{t}$ & transversal wave velocity in the shell \\
\hline $\mathbf{f}_{\mathbf{n}}(s)$ & given column vector \\
\hline$h$ & thickness of the shell \\
\hline$I_{n}$ & modified Bessel functions of order $n$ of the first kind \\
\hline$J_{n}$ & Bessel functions of order $n$ of the first kind \\
\hline$K_{n}$ & modified Bessel functions of order $n$ of the second kind \\
\hline $\mathcal{M}=\rho_{f} / \rho_{s}$ & density ratio \\
\hline $\mathbf{M}_{\mathbf{n}}(s)$ & $4 \times 4$ matrices built from the line vectors $\hat{\mathbf{S}}_{\mathbf{r r}}^{\mathbf{n}}$ and $\hat{\mathbf{S}}_{\mathbf{r} \theta}^{\mathbf{n}}$ \\
\hline$p_{\text {dif }}$ & diffraction pressure \\
\hline$p_{d i f}^{n}$ & Fourier coefficients of the diffraction pressure \\
\hline$p_{\text {ext }}$ & external fluid pressure \\
\hline$p_{\text {inc }}$ & incident pressure \\
\hline$p_{i n c}^{n}$ & Fourier coefficients of the incident pressure \\
\hline$p_{\text {int }}$ & internal fluid pressure \\
\hline$p_{i n t}^{n}$ & Fourier coefficients of the internal fluid pressure \\
\hline$\hat{\mathbf{P}}_{\text {int }}^{\mathrm{n}}$ & line vectors linking $\hat{p}_{i n t}^{n}$ to $\mathbf{a}_{\mathbf{n}}$ \\
\hline$p_{\text {rad }}$ & radiation pressure \\
\hline$p_{\text {rad }}^{n}$ & Fourier coefficients of the radiation pressure \\
\hline$\hat{\mathbf{P}}_{\text {rad }}^{\mathrm{n}}$ & line vectors linking $\hat{p}_{\text {rad }}^{n}$ to $\mathbf{a}_{\mathbf{n}}$ \\
\hline$r$ & radial coordinate of the shell \\
\hline$R$ & radial coordinate of the incident fields \\
\hline$r_{i}$ & inner dimensionless shell radius \\
\hline$R_{s}$ & external radius of the shell \\
\hline$s$ & Laplace variable \\
\hline$\hat{\mathrm{S}}_{\mathrm{rr}}^{\mathrm{n}}$ & line vectors linking $\hat{\sigma}_{r r}^{n}$ to $\mathbf{a}_{\mathbf{n}}$ \\
\hline$\hat{\mathbf{S}}_{\mathbf{r} \theta}^{n}$ & line vectors linking $\hat{\sigma}_{r \theta}^{n}$ to $\mathbf{a}_{\mathbf{n}}$ \\
\hline$t$ & time variable \\
\hline $\mathbf{U}=\left\{U_{r}, U_{\theta}\right\}^{T}$ & vector displacement into the shell \\
\hline$U_{r}$ & radial displacement into the shell \\
\hline$U_{r}^{n}$ & Fourier coefficients of the radial displacement into the shell \\
\hline$U_{\theta}$ & angular displacement into the shell \\
\hline$U_{\theta}^{n}$ & Fourier coefficients of the angular displacement into the shell \\
\hline$V_{\text {inc }}$ & incident velocity field \\
\hline$v_{i n c}^{n}$ & Fourier coefficients of the incident velocity field \\
\hline$Y_{n}$ & Bessel functions of order $n$ of the second kind \\
\hline$\theta$ & angular coordinate \\
\hline$\rho_{f}$ & fluid density \\
\hline$\rho_{s}$ & shell density \\
\hline$\Omega_{l}=c_{f} / c_{l}$ & velocity ratio \\
\hline$\Omega_{t}=c_{f} / c_{t}$ & velocity ratio \\
\hline$\phi, \psi$ & scalar potentials \\
\hline$\phi_{n}, \psi_{n}$ & Fourier coefficients of the scalar potentials \\
\hline$\sigma_{r r}, \sigma_{r \theta}$ & stresses in the shell \\
\hline$\sigma_{r r}^{n}, \sigma_{r \theta}^{n}$ & Fourier coefficients of the stresses \\
\hline$\hat{\Xi}_{n}^{e}$ & complex external transfer function \\
\hline$\hat{\Xi}_{n}^{i}$ & complex internal transfer function \\
\hline
\end{tabular}




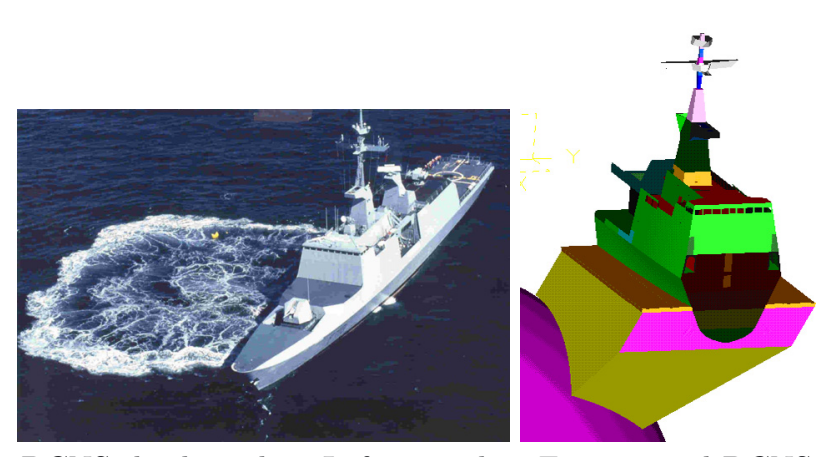

DCNS shock trial on Lafayette-class Frigates and DCNS numerical model of Lafayette-class Frigate

Fig. 1. From full scale shock trial... to numerical simulation.

problems [2]. A full-scale and full-physic modelling of the explosion and ship response is therefore not accessible to the engineer so far, even if such problems tend to be nowadays modelled using whole ship analysis approaches $[3,4]$, see Figure 1.

Many Navies and shipbuilders throughout the world have conducted a series of experiments since the Second World War (from small scale/simple structures to full scale/real structures UNDEX testing [5], see for instance Fig. 1) in order to understand the physics at stake and to gather the test results in databases, which are used for naval ships specifications.

As numerical simulations are expanding in various engineering domains, among which shipbuilding [6], full scale numerical modelling of more and more complex geometries (from ship-like $[7,8]$ to real $[4,9,10]$ ship structures, see Fig. 1) can be performed using dedicated numerical codes, named "hydrocodes" [11-13], whose development is the result of numerous physical and numerical modelling of underwater shock/immersed structure interactions, see for instance Refs. [14-18]. In order to be numerically efficient for industrial applications, fluidstructure interaction is described in these codes through asymptotic theories $[19,20]$, such as the so-called "Doubly Asymptotic Approximation" (DAA, see for instance Refs. $[21,22])$. This approach provides a description of FSI which is physically accurate in the low and high frequency ranges.

On the one hand, full scale shock experiments provide "exact" measurements of fluid-structure interaction phenomenon on real and complex structures, but are very costly from the practical point of view; on the other hand, full scale shock simulations provide "simplified" descriptions of fluid-structure interaction phenomenon on virtual complex structures, but are very costly from the numerical point of view. From the engineering standpoint, there still exists a need for intermediate approaches, e.g. for "exact" solutions on "simplified" models of fluid-structure interaction in shock wave/immersed structure problems.

The purpose of the present paper is precisely to investigate such an intermediate approach: as a matter of fact, building a simplified model allows the engineer on the one hand to understand the physics of fluid-structure coupling in shock wave/immersed structure interactions and on the other hand to derive some input data for shock analysis in pre-design studies.

The transient dynamics of evacuated and fluid-filled circular elastic shells, submerged in an infinite fluid medium and subjected to an external weak shock wave, is considered here. This geometrically simple fluid-structure problem can be representative of several important applications, from underwater pipelines to submarines, which makes its study of definite practical interest. In addition to its industrial applications, this set-up is of academic concern since it illustrates in a closed form the complex coupling between the acoustic phenomena in the fluid and the elastic phenomena in the structure, including the shock transparency of the shell, the reflection and focusing of the internal acoustic wave and the radiation into the fluid of elastic waves propagating in the shell.

While the circular shell/acoustic medium interaction problem has already been tackled in the frequency domain with a full elastic model $[23,24]$, the purely transient case has only been achieved with simplified thin shell models based on the Love-Kirchhoff hypotheses for the structural dynamics, see references [25-29] for an evacuated shell and references $[15,16,30]$ for a fluid-filled one. With this structural model, the resulting radiated pressure field displays some discrepancies related to the $A_{0} / S_{0}$ waves when compared to the experimental data available in the literature [31-33]. Since the thin shell models are known to be restricted to the low frequency domain (the wavelengths in the structure and in the fluid must be larger than the shell thickness), and a weak shock wave may contain some high frequency components, the discrepancies should be overcome by the use of a full elastic model. This is done here in a two-dimensional framework. The approach is semi-analytical and based on the methods of Laplace transform in time, Fourier series expansions and separation of variables in space.

\section{Shell dynamics}

Let us consider a two-dimensional elastic shell of constant thickness $h$, external radius $R_{s}$, density $\rho_{s}$ and longitudinal and transversal waves velocities $c_{l}$ and $c_{t}$, respectively. It is submerged in an infinite fluid medium of density $\rho_{f}$ and subjected to a weak incident acoustic excitation. It can also be filled by an internal fluid of the same density as the external one. The displacements of the shell are assumed sufficiently small compared to both its radius and thickness, so that the boundary conditions can be applied on the shell boundaries at rest. A schematic of the problem is shown in Figure 2. In the following, the variables are written in a dimensionless form: the lengths are normalized by $R_{s}$, the time by $R_{s} / c_{f}$ with $c_{f}$ being the sound speed in the fluid, and the pressure by $\rho_{f} c_{f}^{2}$.

The displacements $\mathbf{U}$ into the shell are expressed in the two-dimensional theory of elasticity by the scalar potentials $\phi$ and $\psi$ such that

$$
\mathbf{U}=\nabla \phi+\nabla \times\left(\psi \mathbf{e}_{\mathbf{z}}\right)
$$




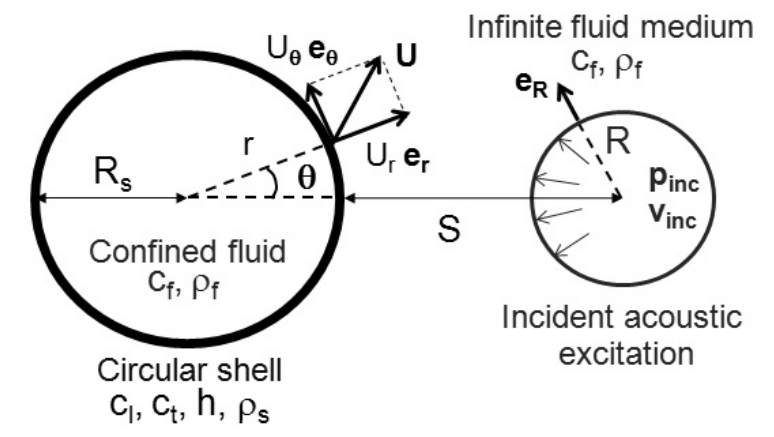

Fig. 2. Submerged two-dimensional circular cylindrical shell subjected to an incident acoustic excitation.

The displacement potentials satisfy the dimensionless wave equations in the Laplace domain:

$$
\begin{aligned}
\nabla^{2} \hat{\phi}-s^{2} \Omega_{l}^{2} \hat{\phi} & =0 \\
\nabla^{2} \hat{\psi}-s^{2} \Omega_{t}^{2} \hat{\psi} & =0
\end{aligned}
$$

where the hats denote the Laplace transforms of the corresponding quantities and $s$ is the Laplace variable. $\Omega_{l}$ and $\Omega_{t}$ are respectively given by $\Omega_{l}=c_{f} / c_{l}$ and $\Omega_{t}=c_{f} / c_{t}$. Since the circular shell is assumed elastic, the constraints are linearly linked to the displacements $\mathbf{U}=\left\{U_{r}, U_{\theta}\right\}^{T}$ by the Hooke law (here in a dimensionless form):

$$
\begin{aligned}
& \hat{\sigma}_{r r}=\frac{1}{\mathcal{M} \Omega_{l}^{2}} \frac{\partial \hat{U}_{r}}{\partial r}+\frac{1}{\mathcal{M}}\left(\frac{1}{\Omega_{l}^{2}}-\frac{2}{\Omega_{t}^{2}}\right)\left(\frac{\hat{U}_{r}}{r}+\frac{1}{r} \frac{\partial \hat{U}_{\theta}}{\partial \theta}\right) \\
& \hat{\sigma}_{r \theta}=\frac{1}{\mathcal{M} \Omega_{t}^{2}}\left(\frac{1}{r} \frac{\partial \hat{U}_{r}}{\partial \theta}-\frac{\hat{U}_{\theta}}{r}+\frac{\partial \hat{U}_{\theta}}{\partial r}\right)
\end{aligned}
$$

with $\mathcal{M}=\rho_{f} / \rho_{s}$ the density ratio. The dimensionless boundary conditions are given by

$$
\begin{aligned}
\hat{\sigma}_{r r}(1, \theta, s) & =-\hat{p}_{\text {ext }}(1, \theta, s) \\
\hat{\sigma}_{r r}\left(r_{i}, \theta, s\right) & =\hat{p}_{\text {int }}\left(r_{i}, \theta, s\right) \\
\hat{\sigma}_{r \theta}(1, \theta, s) & =0 \\
\hat{\sigma}_{r \theta}\left(r_{i}, \theta, s\right) & =0
\end{aligned}
$$

where $r_{i}=1-h / R_{s}$ is the inner dimensionless shell radius, $p_{\text {ext }}$ the external fluid pressure and $p_{\text {int }}$ the internal one. In order to solve this elastic shell-acoustic fields interaction problem, the quantities $\hat{\sigma}_{r r}$ and $\hat{\sigma}_{r \theta}$ in the boundary conditions have first to be expressed in terms of the potentials displacements $\phi$ and $\psi$.
To achieve this goal, it can be useful to perform the following Fourier series expansions:

$$
\begin{aligned}
\hat{\phi}(r, \theta, s) & =\sum_{n=0}^{\infty} \hat{\phi}_{n}(r, s) \cos (n \theta) \\
\hat{\psi}(r, \theta, s) & =\sum_{n=1}^{\infty} \hat{\psi}_{n}(r, s) \sin (n \theta) \\
\hat{U}_{r}(r, \theta, s) & =\sum_{n=0}^{\infty} \hat{U}_{r}^{n}(r, s) \cos (n \theta) \\
\hat{U}_{\theta}(r, \theta, s) & =\sum_{n=1}^{\infty} \hat{U}_{\theta}^{n}(r, s) \sin (n \theta) \\
\mathcal{M} \hat{\sigma}_{r r}(r, \theta, s) & =\sum_{n=0}^{\infty} \hat{\sigma}_{r r}^{n}(r, s) \cos (n \theta) \\
\mathcal{M} \hat{\sigma}_{r \theta}(r, \theta, s) & =\sum_{n=1}^{\infty} \hat{\sigma}_{r \theta}^{n}(r, s) \sin (n \theta)
\end{aligned}
$$

for $r \in\left[r_{i}, 1\right]$. By solving the wave equations (2), with the method of separation of variables, the potentials components in equations $(5,6)$ can be written

$$
\begin{aligned}
& \hat{\phi}_{n}(r, s)=A_{n}(s) J_{n}\left(i \Omega_{l} s r\right)+B_{n}(s) Y_{n}\left(i \Omega_{l} s r\right) \\
& \hat{\psi}_{n}(r, s)=C_{n}(s) J_{n}\left(i \Omega_{t} s r\right)+D_{n}(s) Y_{n}\left(i \Omega_{t} s r\right)
\end{aligned}
$$

where $J_{n}$ and $Y_{n}$ denote the Bessel functions of order $n$ of the first and second kind respectively [34]. The factors $A_{n}$, $B_{n}, C_{n}$ and $D_{n}$ are the unknown coefficients which have to be determined with the boundary conditions on the cylindrical shell. It is convenient for the following to group the unknown factors in the column vector $\mathbf{a}_{\mathbf{n}}$ such as

$$
\mathbf{a}_{\mathbf{n}}(s)=\left\{A_{n}(s), B_{n}(s), C_{n}(s), D_{n}(s)\right\}^{T}
$$

Then the substitution of the Fourier series expansions for the potentials and displacements fields (5-8), into equations (1) and the use of equation (11) yield the displacements coefficients as functions of the problem unknowns:

$$
\begin{aligned}
\hat{U}_{r}^{n}(r, s)= & \frac{1}{r}\left[n J_{n}\left(k_{l}\right)-k_{l} J_{n+1}\left(k_{l}\right)\right] A_{n}(s) \\
& +\frac{1}{r}\left[n Y_{n}\left(k_{l}\right)-k_{l} Y_{n+1}\left(k_{l}\right)\right] B_{n}(s) \\
& +\frac{n}{r} J_{n}\left(k_{t}\right) C_{n}(s)+\frac{n}{r} Y_{n}\left(k_{t}\right) D_{n}(s) \\
\hat{U}_{\theta}^{n}(r, s)= & -\frac{n}{r} J_{n}\left(k_{l}\right) A_{n}(s)-\frac{n}{r} Y_{n}\left(k_{l}\right) B_{n}(s) \\
& -\frac{1}{r}\left[n J_{n}\left(k_{t}\right)-k_{t} J_{n+1}\left(k_{t}\right)\right] C_{n}(s) \\
& -\frac{1}{r}\left[n Y_{n}\left(k_{t}\right)-k_{t} Y_{n+1}\left(k_{t}\right)\right] D_{n}(s)
\end{aligned}
$$

where the coefficients $k_{l}$ et $k_{t}$ are given by $k_{l}=i \Omega_{l} s r$ and $k_{t}=i \Omega_{t} s r$. In order to write the quantities $\sigma_{r r}$ and $\sigma_{r \theta}$ in terms of the problem unknowns, the Fourier series expansions (7-10) are then substituted into the Hooke 
law (3), and the above relations for the displacements coefficients are used, which yield:

$$
\begin{aligned}
& \hat{\sigma}_{r r}^{n}(r, s)=\hat{\mathbf{S}}_{\mathbf{r r}}^{\mathbf{n}}(r, s) \mathbf{a}_{\mathbf{n}}(s) \\
& \hat{\sigma}_{r \theta}^{n}(r, s)=\hat{\mathbf{S}}_{\mathbf{r} \theta}^{\mathbf{n}}(r, s) \mathbf{a}_{\mathbf{n}}(s)
\end{aligned}
$$

where the quantities $\hat{\mathbf{S}}_{\mathbf{r r}}^{\mathbf{n}}$ and $\hat{\mathbf{S}}_{\mathbf{r} \theta}^{\mathbf{n}}$ are line vectors whose coefficients are explicitly given in Appendix. The left-hand side terms of the boundary conditions (4) are now fully determined. In order to solve the fluid-structure interaction problem, the pressure on the internal and external boundaries of the shell are still to be expressed as functions of the problem unknowns.

\section{Fluid dynamics}

We assume that the shell is submerged into (and filled with) linearly compressible, irrotational and inviscid fluid. The internal and external dimensionless fluid pressures, respectively $p_{\text {int }}$ and $p_{\text {ext }}$, are therefore governed by the wave equations in the Laplace domain:

$$
\begin{aligned}
\nabla^{2} \hat{p}_{\text {int }}-s^{2} \hat{p}_{\text {int }} & =0 \\
\nabla^{2} \hat{p}_{\text {ext }}-s^{2} \hat{p}_{\text {ext }} & =0
\end{aligned}
$$

By virtue of the acoustic problem linearity, the external pressure field $p_{\text {ext }}$ is classically divided into three components: the incident pressure $p_{\text {inc }}$, the diffraction pressure $p_{\text {dif }}$ and the external radiation pressure $p_{\text {rad }}$,

$$
p_{\text {ext }}=p_{\text {inc }}+p_{\text {dif }}+p_{\text {rad }}
$$

The incident pressure $p_{i n c}$ is a given data, described in Appendix for a weak shock wave. The diffraction pressure $p_{\text {dif }}$ is introduced in order to balance the normal component of the given incident wave velocity on the body surface, $\mathbf{v}_{\mathbf{i n c}} \cdot \mathbf{e}_{\mathbf{r}}$. The last component $p_{\text {rad }}$, represents the pressure radiated by the deformations of the submerged body. Hence $p_{\text {dif }}$ and $p_{\text {rad }}$ satisfy the wave equations, with the following boundary conditions in the Laplace domain:

$$
\begin{aligned}
& \left.\frac{\partial \hat{p}_{d i f}}{\partial r}\right|_{r=1}=\left.s \hat{\mathbf{v}}_{\mathbf{i n c}}\right|_{r=1} \cdot \mathbf{e}_{\mathbf{r}}, \quad \lim _{r \rightarrow \infty} \hat{p}_{d i f}=0 \\
& \left.\frac{\partial \hat{p}_{\text {rad }}}{\partial r}\right|_{r=1}=-s^{2} \hat{U}_{r}(1, \theta, s), \quad \lim _{r \rightarrow \infty} \hat{p}_{r a d}=0
\end{aligned}
$$

As for the internal pressure, $p_{\text {int }}$, it is only induced by the deformations of the shell internal boundary and therefore satisfies the following boundary conditions:

$$
\left.\frac{\partial \hat{p}_{\text {int }}}{\partial r}\right|_{r=r_{i}}=s^{2} \hat{U}_{r}\left(r_{i}, \theta, s\right), \quad\left|\hat{p}_{\text {int }}\right|_{r=0} \mid<\infty
$$

Due to the circular geometry of the shell, the pressure fields and the normal component of the incident wave velocity can be decomposed into the Fourier series expansions:

$$
\begin{aligned}
\hat{p}_{\text {inc }}(1, \theta, s) & =\sum_{n=0}^{\infty} \hat{p}_{\text {inc }}^{n}(s) \cos (n \theta) \\
\hat{p}_{\text {dif }}(r, \theta, s) & =\sum_{n=0}^{\infty} \hat{p}_{\text {dif }}^{n}(r, s) \cos (n \theta) \text { for } r \geq 1 \\
\hat{p}_{\text {rad }}(r, \theta, s) & =\sum_{n=0}^{\infty} \hat{p}_{\text {rad }}^{n}(r, s) \cos (n \theta) \text { for } r \geq 1 \\
\hat{p}_{\text {int }}(r, \theta, s) & =\sum_{n=0}^{\infty} \hat{p}_{\text {int }}^{n}(r, s) \cos (n \theta) \text { for } r \leq r_{i} \\
\hat{\mathbf{v}}_{\mathbf{i n c}}(1, \theta, s) \cdot \mathbf{e}_{\mathbf{r}} & =\sum_{n=0}^{\infty} \hat{v}_{\text {inc }}^{n}(s) \cos (n \theta)
\end{aligned}
$$

By solving the wave equations for the diffracted, radiated and internal pressure fields with the method of separation of variables, and by introducing the Fourier series expansions (7, 23-26) into the boundary conditions (1921 ), the Fourier components of the pressure fields can be expressed as functions of the given quantities and shell displacements:

$$
\begin{aligned}
& \hat{p}_{d i f}^{n}(r, s)=-\hat{\Xi}_{n}^{e}(r, s) s \hat{v}_{i n c}^{n}(s) \\
& \hat{p}_{r a d}^{n}(r, s)=\hat{\Xi}_{n}^{e}(r, s) s^{2} \hat{U}_{r}^{n}(1, s) \\
& \hat{p}_{\text {int }}^{n}(r, s)=-\hat{\Xi}_{n}^{i}(r, s) s^{2} \hat{U}_{r}^{n}\left(r_{i}, s\right)
\end{aligned}
$$

where the complex transfer functions are given by

$$
\begin{aligned}
& \hat{\Xi}_{n}^{e}(r, s)=\frac{K_{n}(r s)}{s K_{n+1}(s)-n K_{n}(s)} \quad \text { for } \quad r \geq 1 \\
& \hat{\Xi}_{n}^{i}(r, s)=\frac{I_{n}(r s)}{s I_{n+1}(s)+n I_{n}(s)} \quad \text { for } \quad r \leq r_{i}
\end{aligned}
$$

with $I_{n}$ and $K_{n}$ the modified Bessel functions of order $n$ of the first and second kind respectively [34]. Then the substitution of the displacements coefficients $(13,14)$ into equations $(28,29)$ yields the radiated and internal pressure fields components in terms of the problem unknowns:

$$
\begin{aligned}
& \hat{p}_{\text {rad }}^{n}(r, s)=\hat{\mathbf{P}}_{\text {rad }}^{\mathbf{n}}(r, s) \mathbf{a}_{\mathbf{n}}(s) \\
& \hat{p}_{\text {int }}^{n}(r, s)=\hat{\mathbf{P}}_{\text {int }}^{\mathbf{n}}(r, s) \mathbf{a}_{\mathbf{n}}(s)
\end{aligned}
$$

where the quantities $\hat{\mathbf{P}}_{\text {rad }}^{\mathbf{n}}$ and $\hat{\mathbf{P}}_{\text {int }}^{\mathbf{n}}$ are line vectors whose coefficients are given in Appendix.

\section{Resolution of the fluid-structure interaction problem}

The left and right-hand sides of the boundary conditions (4) are now fully determined. The substitution of the Fourier series expansions $(9,10,22-25)$ and relations $(15,27,31)$ into these boundary conditions yields a 
linear algebraic system of size $4 \times 4$ for each Fourier mode, involving the problem unknowns and the given data only:

$$
\mathbf{M}_{\mathbf{n}}(s) \mathbf{a}_{\mathbf{n}}(s)=\mathbf{f}_{\mathbf{n}}(s)
$$

with the matrix $\mathbf{M}_{\mathbf{n}}(s)$ built from the line vectors $(35,36)$ such as

$$
\mathbf{M}_{\mathbf{n}}(s)=\left[\begin{array}{c}
\hat{\mathbf{S}}_{\mathbf{r r}}^{\mathbf{n}}(1, s) \\
\hat{\mathbf{S}}_{\mathbf{r r}}^{\mathbf{n}}\left(r_{i}, s\right) \\
\hat{\mathbf{S}}_{\mathbf{r} \theta}^{\mathbf{n}}(1, s) \\
\hat{\mathbf{S}}_{\mathbf{r} \theta}^{\mathbf{n}}\left(r_{i}, s\right)
\end{array}\right]+\mathcal{M}\left[\begin{array}{c}
\hat{\mathbf{P}}_{\mathbf{r a d}}^{\mathbf{n}}(1, s) \\
-\hat{\mathbf{P}}_{\mathbf{i n t}}^{\mathbf{n}}\left(r_{i}, s\right) \\
\mathbf{0} \\
\mathbf{0}
\end{array}\right]
$$

The given column vector $\mathbf{f}_{\mathbf{n}}(s)$ represents the incident and diffracted pressure fields on the shell:

$$
\mathbf{f}_{\mathbf{n}}(s)=-\mathcal{M}\left\{\hat{p}_{i n c}^{n}(s)-\hat{\Xi}_{n}^{e}(1, s) s \hat{v}_{i n c}^{n}(s), 0,0,0\right\}^{T}
$$

The solution is straightforward: it consists of simple matrix inversions, $\mathbf{a}_{\mathbf{n}}(s)=\mathbf{M}_{\mathbf{n}}{ }^{-1}(s) \mathbf{f}_{\mathbf{n}}(s)$, for each point of the Laplace variable and Fourier mode, evaluation of the Fourier coefficients of the quantities, numerical inversions of the Laplace transforms [35] and Fourier series summations.

\section{Results}

The method is illustrated by computing the shell response to a weak underwater explosion, which corresponds to the detonation of $1 \mathrm{~kg}$ of TNT located at a dimensionless stand-off of $S=2$. The derivation of the induced incident pressure and velocity fields required for the fluid-structure interaction problem, respectively $\hat{p}_{\text {inc }}(1, \theta, s)$ and $\hat{\mathbf{v}}_{\text {inc }}(1, \theta, s) \cdot \mathbf{e}_{\mathbf{r}}$, is described in Appendix. In the following, 120 terms are kept in the Fourier series expansions. It is shown in reference [29] that this number provides converged solutions for the shell displacements and radiated pressure, but is not sufficient for the diffracted one, for which even 300 terms result in noticeable local errors.

\subsection{Illustrations without internal fluid}

The resulting pressure field in the fluid domain is illustrated in Figure 3a for a shell of thickness $h / R_{s}=0.03$ (with material parameters $\rho_{s}=7800 \mathrm{~kg} \cdot \mathrm{m}^{-3}, c_{l}=$ $5800 \mathrm{~m} . \mathrm{s}^{-1}, c_{t}=3100 \mathrm{~m} . \mathrm{s}^{-1}, \rho_{f}=1000 \mathrm{~kg} \cdot \mathrm{m}^{-3}$ and $\left.c_{f}=1470 \mathrm{~m} . \mathrm{s}^{-1}\right)$. The pressure field derived with a thin shell model [28] is shown in Figure 3b.

The incident $(I)$ and diffracted $(D)$ waves are obviously the same since they are independent of the shell dynamics. Some discrepancies arise for the shell-induced waves, i.e. the pseudo-Rayleigh wave $\left(A_{0}\right)$ and the Lamb wave $\left(S_{0}\right)$. With the elastic shell model, these two waves are well distinguished and agree with experimental data provided by Derbesse et al. [33]. As for the pressure field

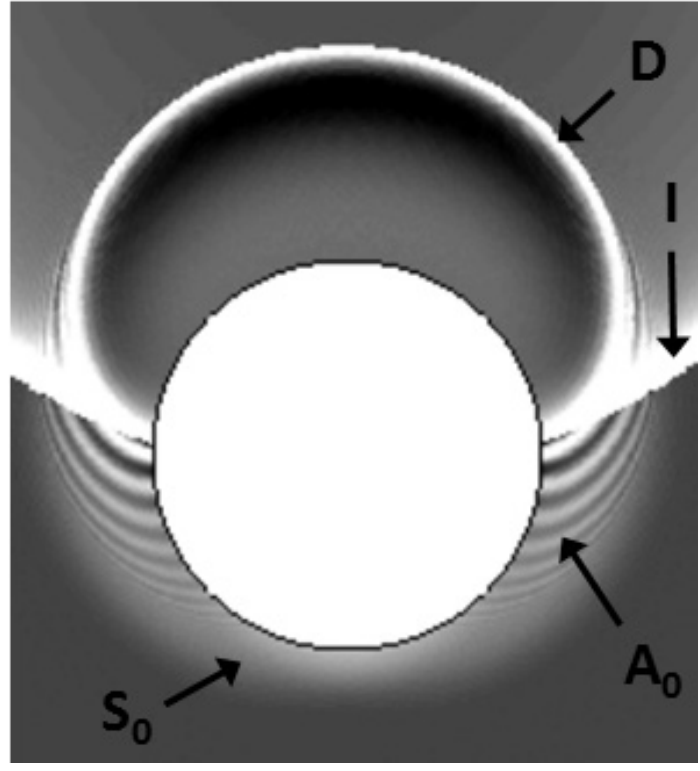

(a)

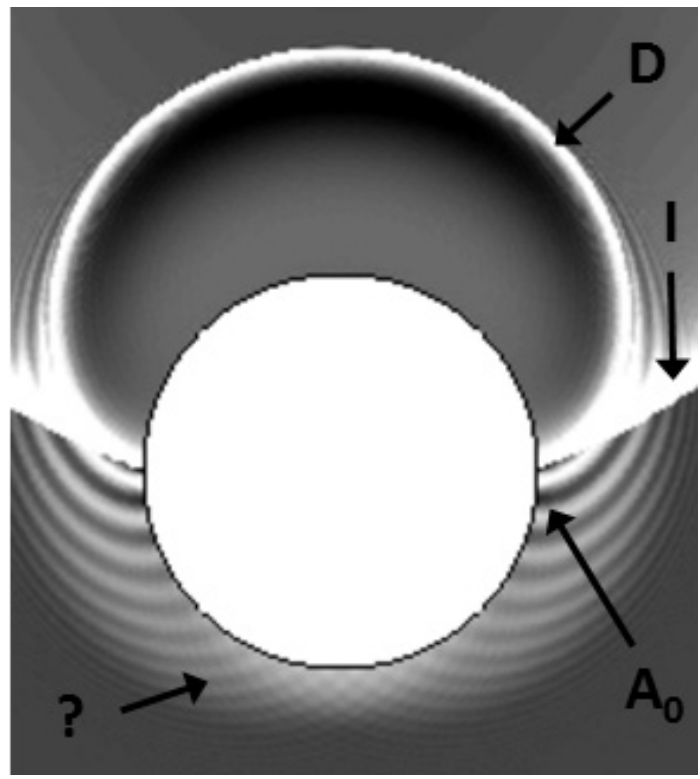

(b)

Fig. 3. Pressure fields in the fluid domain for $h / R_{s}=0.03$ obtained (a) with the elastic model for the shell dynamics, (b) with the thin shell model based on the Love-Kirchhoff hypotheses [28].

obtained with the thin shell model, the streaky pattern induced by the symmetric $A_{0}$ can be recognized just ahead the incident wave, but the $S_{0}$ and $A_{0}$ waves cannot in this case be identified. While the $S_{0}$ wave seems to be well represented by the thin shell model, the discrepancies arise from the $A_{0}$ one: its group velocity is correct, but the phase velocity of its high frequency components is strongly overestimated. The weaknesses of the thin shell model in this case can be explained by computing the power spectrum of the incident pressure; it can be inferred 
from it that more than $10 \%$ of the incident wave energy is outside the validity domain of the thin shell model (which is $2 \pi f / c_{f} \leq 1$ with $f$ the frequencies contained in the incident wave).

\subsection{Illustrations with an internal fluid}

The snapshots of the internal and external radiated pressure fields obtained with the elastic model (with the same parameters as those used in the previous paragraph) are now displayed in Figures 4 and 5. An internal shock wave is seen to propagate through the internal fluid, which illustrates the shock transparency of the elastic shell, a phenomenon already described in the case of a thin shell model [15]. Head waves into the external and internal fluid domains induced by the radiation of elastic waves propagating in the shell can also be seen. As for the evacuated case, $S_{0}$ and $A_{0}$ can be easily identified, particularly in Figure $4 \mathrm{~b}$. The $S_{0}$ waves are induced by the outward motion of the shell surface, resulting in a positive pressure in the external fluid domain and a negative pressure in the internal one. The anti-symmetrical nature of the $A_{0}$ waves is also evident just ahead the incident shock wave in Figures 4b, 4c, since a negative (respectively positive) pressure in the external pressure can be associated to a positive (respectively negative) pressure in the internal one. Another phenomenon can be identified in Figures 4c and $5 \mathrm{a}, 5 \mathrm{~b}$ : the $A_{0}$ wave seems to divide itself into two parts. The first one just ahead the incident shock wave remains anti-symmetrical, and the other one with a higher group velocity seems to be induced by the propagation of a symmetrical wave in the shell. It is well-known in the harmonic regime [36] that a new wave, called $A$-wave, can arise from the fluid loading on the shell. This may be this wave which is observed here. This point deserves more attention and will be the subject of a future work.

\section{Conclusion}

A semi-analytical method to solve the circular elastic submerged shell/weak shock wave interaction problem is proposed. The shell may be evacuated or fluid-filled. For the evacuated case, it is shown that the previously reported drawbacks related to the $A_{0} / S_{0}$ waves are eliminated and the new approach results in much more realistic images of the radiated acoustic field when compared to experiments. For the fluid-filled case, the transient thick shell-weak shock wave interaction problem is explored and the radiated acoustic field is described. The analysis of the stresses in the shell will be the subject of a future work.

Acknowledgements. The first author gratefully acknowledges the financial support provided by the company DCNS Propulsion when he was a PhD student. The second author acknowledges financial support of the Killam Trusts and the Natural Sciences and Engineering Research Council of Canada.

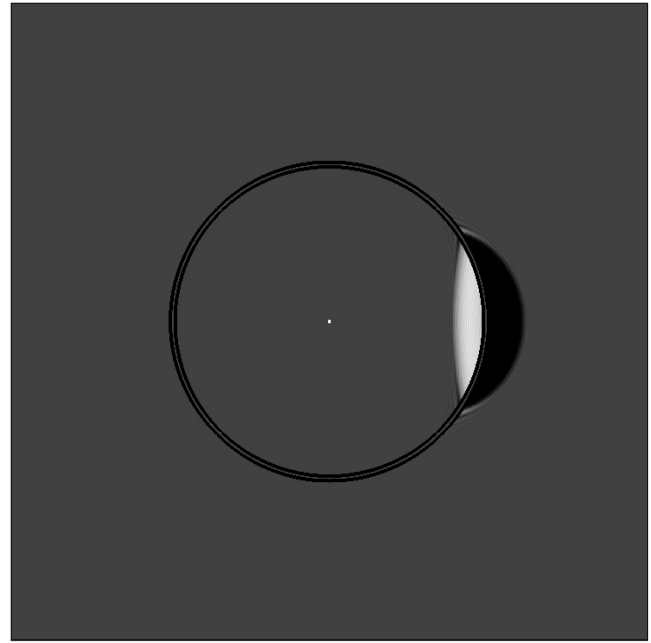

(a) $t=0.2$

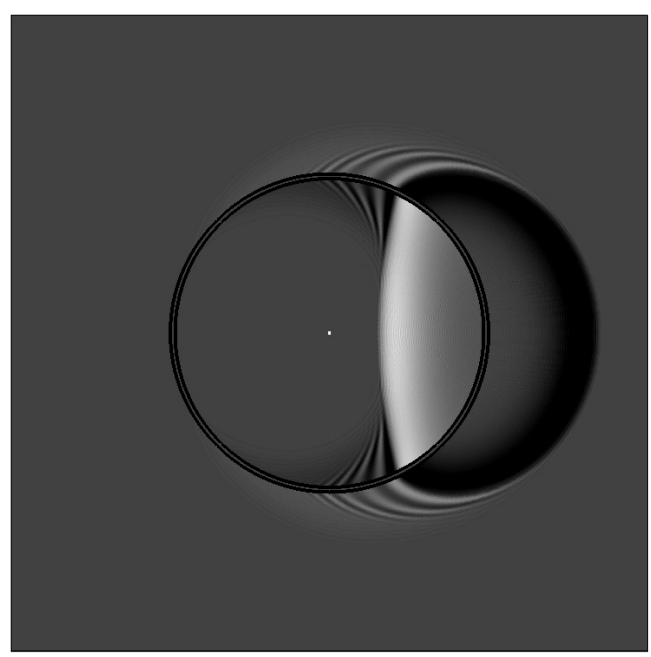

(b) $t=0.7$

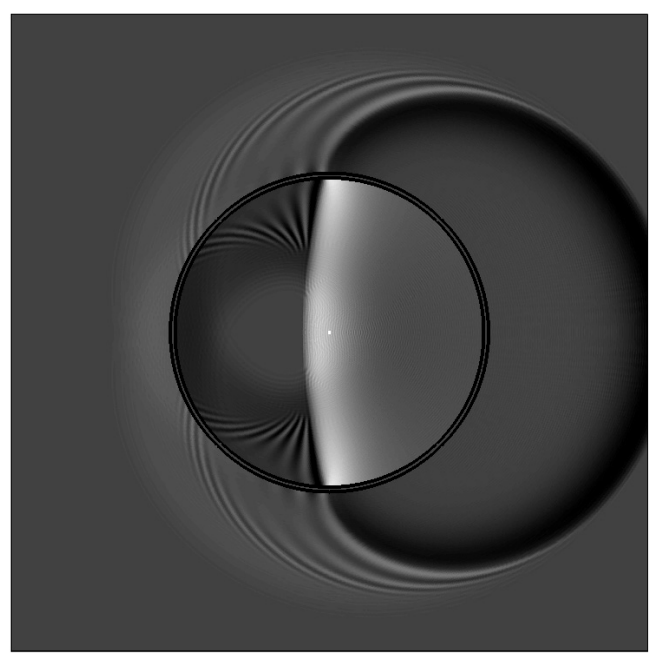

(c) $t=1.2$

Fig. 4. Snapshots of the radiated pressure fields in the external and internal fluid domains obtained with the elastic model for the fluid-filled shell with $h / R_{s}=0.03$, for $t=0.2$ to $t=1.2$. 


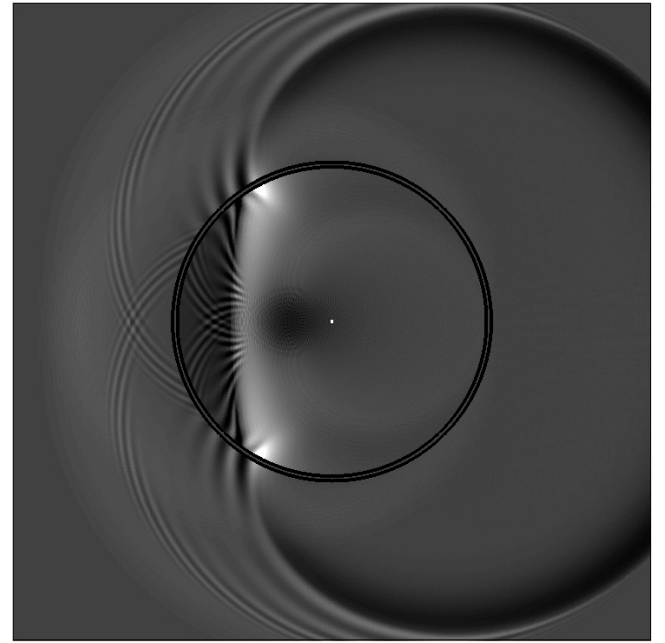

(a) $t=1.6$

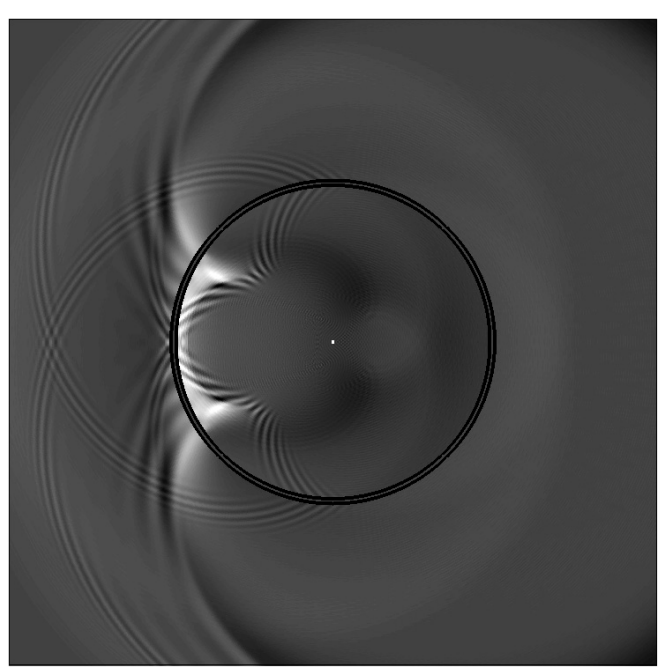

(b) $t=2.1$

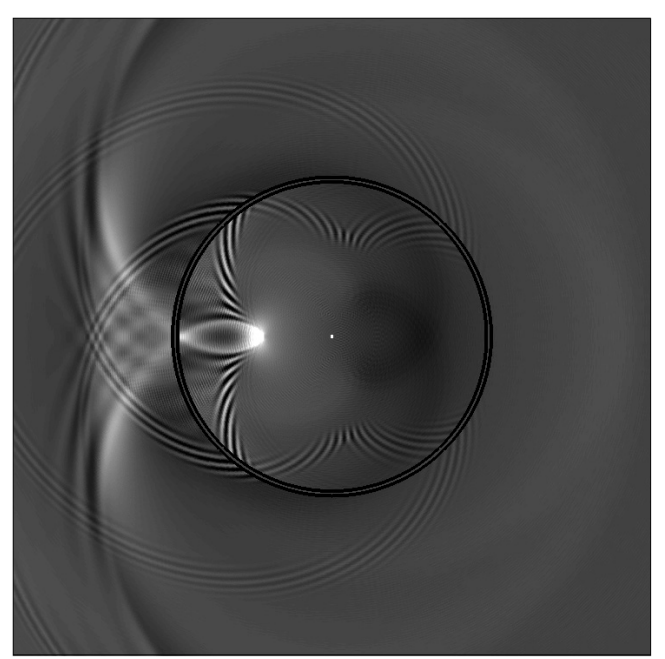

(c) $t=2.6$

Fig. 5. Snapshots of the radiated pressure fields in the external and internal fluid domains obtained with the elastic model for the fluid-filled shell with $h / R_{s}=0.03$, for $t=1.6$ to $t=2.6$.

\section{Appendix}

A. Expressions of the quantities $\hat{\mathrm{S}}_{\mathrm{rr}}^{\mathrm{n}}, \hat{\mathrm{S}}_{\mathrm{r} \theta}^{\mathrm{n}}, \hat{\mathrm{P}}_{\mathrm{rad}}^{\mathrm{n}}$ and $\hat{\mathrm{P}}_{\mathrm{int}}^{\mathrm{n}}$

The quantities $\hat{\mathbf{S}}_{\mathbf{r r}}^{\mathbf{n}}$ and $\hat{\mathbf{S}}_{\mathbf{r} \theta}^{\mathbf{n}}$ appearing in equation (15) are line vectors whose coefficients $\hat{S}_{r r, i}^{n}$ and $\hat{S}_{r \theta, i}^{n}$, for $i=$ $1 \cdots 4$, are explicitly given by

$$
\begin{aligned}
\hat{S}_{r r, 1}^{n}(r, s)= & \frac{2}{\Omega_{t}^{2} r^{2}}\left[n(n-1) J_{n}\left(k_{l}\right)+k_{l} J_{n+1}\left(k_{l}\right)\right] \\
& -\frac{k_{l}^{2}}{\Omega_{l}^{2} r^{2}} J_{n}\left(k_{l}\right) \\
\hat{S}_{r r, 2}^{n}(r, s)= & \frac{2}{\Omega_{t}^{2} r^{2}}\left[n(n-1) Y_{n}\left(k_{l}\right)+k_{l} Y_{n+1}\left(k_{l}\right)\right] \\
& -\frac{k_{l}^{2}}{\Omega_{l}^{2} r^{2}} Y_{n}\left(k_{l}\right) \\
\hat{S}_{r r, 3}^{n}(r, s)= & \frac{2 n}{\Omega_{t}^{2} r^{2}}\left[(n-1) J_{n}\left(k_{t}\right)-k_{t} J_{n+1}\left(k_{t}\right)\right] \\
\hat{S}_{r r, 4}^{n}(r, s)= & \frac{2 n}{\Omega_{t}^{2} r^{2}}\left[(n-1) Y_{n}\left(k_{t}\right)-k_{t} Y_{n+1}\left(k_{t}\right)\right] \\
\hat{S}_{r \theta, 1}^{n}(r, s)= & -\frac{2 n}{\Omega_{t}^{2} r^{2}}\left[(n-1) J_{n}\left(k_{l}\right)-k_{l} J_{n+1}\left(k_{l}\right)\right] \\
\hat{S}_{r \theta, 2}^{n}(r, s)= & -\frac{2 n}{\Omega_{t}^{2} r^{2}}\left[(n-1) Y_{n}\left(k_{l}\right)-k_{l} Y_{n+1}\left(k_{l}\right)\right] \\
\hat{S}_{r \theta, 3}^{n}(r, s)= & -\frac{1}{\Omega_{t}^{2} r^{2}}\left[\left(2 n(n-1)-k_{t}^{2}\right) J_{n}\left(k_{t}\right)\right. \\
& \left.+2 k_{t} J_{n+1}\left(k_{t}\right)\right] \\
\hat{S}_{r \theta, 4}^{n}(r, s)= & -\frac{1}{\Omega_{t}^{2} r^{2}}\left[\left(2 n(n-1)-k_{t}^{2}\right) Y_{n}\left(k_{t}\right)\right. \\
& \left.+2 k_{t} Y_{n+1}\left(k_{t}\right)\right]
\end{aligned}
$$

As for the quantities $\hat{\mathbf{P}}_{\text {rad }}^{\text {n }}$ and $\hat{\mathbf{P}}_{\text {int }}^{\text {n }}$ in equation (31), they are also line vectors, whose coefficients $\hat{P}_{r a d, i}^{n}$ and $\hat{P}_{i n t, i}^{n}$, for $i=1 \cdots 4$, can be written

$$
\begin{aligned}
\hat{P}_{r a d, 1}^{n}(r, s) & =\left[n J_{n}\left(k_{l}\right)-k_{l} J_{n+1}\left(k_{l}\right)\right] s^{2} \hat{\Xi}_{n}^{e}(r, s) \\
\hat{P}_{r a d, 2}^{n}(r, s) & =\left[n Y_{n}\left(k_{l}\right)-k_{l} Y_{n+1}\left(k_{l}\right)\right] s^{2} \hat{\Xi}_{n}^{e}(r, s) \\
\hat{P}_{r a d, 3}^{n}(r, s) & =n J_{n}\left(k_{t}\right) s^{2} \hat{\Xi}_{n}^{e}(r, s) \\
\hat{P}_{r a d, 4}^{n}(r, s) & =n Y_{n}\left(k_{t}\right) s^{2} \hat{\Xi}_{n}^{e}(r, s) \\
\hat{P}_{i n t, 1}^{n}(r, s) & =-\frac{1}{r_{i}}\left[n J_{n}\left(k_{l}\right)-k_{l} J_{n+1}\left(k_{l}\right)\right] s^{2} \hat{\Xi}_{n}^{i}(r, s) \\
\hat{P}_{i n t, 2}^{n}(r, s) & =-\frac{1}{r_{i}}\left[n Y_{n}\left(k_{l}\right)-k_{l} Y_{n+1}\left(k_{l}\right)\right] s^{2} \hat{\Xi}_{n}^{i}(r, s) \\
\hat{P}_{i n t, 3}^{n}(r, s) & =-\frac{n}{r_{i}} J_{n}\left(k_{t}\right) s^{2} \hat{\Xi}_{n}^{i}(r, s) \\
\hat{P}_{i n t, 4}^{n}(r, s) & =-\frac{n}{r_{i}} Y_{n}\left(k_{t}\right) s^{2} \hat{\Xi}_{n}^{i}(r, s)
\end{aligned}
$$

\section{B. The incident weak shock wave}

In order to solve the fluid-structure interaction problem, the incident pressure and velocity fields, respectively 
$\hat{p}_{\text {inc }}(1, \theta, s)$ and $\hat{\mathbf{v}}_{\text {inc }}(1, \theta, s) \cdot \mathbf{e}_{\mathbf{r}}$, induced by a weak underwater explosion have to be expressed. For this purpose, the similitude relation for the far-field shock-wave pressure profile provided by Price [37] is used. It consists of a hyper-acoustic pulse which, as in reference [38], is assumed to propagate in the far-field at the linear acoustic speed of sound:

$$
\begin{aligned}
P_{\text {inc }}(R, t) & =P_{c}\left[\frac{a_{c}}{R}\right]^{1+A} F\left(\left[\frac{a_{c}}{R}\right]^{B} \frac{\tau}{T_{c}}\right) H(\tau) \\
\tau & =t-(R-S) / c_{f}, \quad T_{c}=\frac{a_{c}}{\nu_{c}}
\end{aligned}
$$

with $R$ the distance from the explosive charge of radius $a_{c}$ and $S$ the stand-off, as illustrated in Figure 2. $H$ denotes the Heaviside step function and $F$ a known function given by $F(t)=0.8251 \mathrm{e}^{-1.338 t}+0.1749 \mathrm{e}^{-0.1805 t}$ valid for $t \leq 7$. The constants depend on the explosive and take the following values for the TNT: $P_{c}=1.67 \mathrm{GPa}$, $\nu_{c}=1010 \mathrm{~m} \cdot \mathrm{s}^{-1}, A=0.18$ and $B=0.185$. The incident velocity field associate with the pressure profile of this spherical shock wave is deduced through acoustic relations as in reference [38]:

$$
\begin{aligned}
\mathbf{V}_{\text {inc }}(R, t)= & \left\{\frac{P_{i n c}(R, t)}{\rho_{f} c_{f}}+\frac{1+A}{\rho_{f} R} \int_{0}^{\tau} P_{i n c}(R, \xi) \mathrm{d} \xi\right. \\
& \left.+\frac{1}{T_{c}} \frac{B}{\rho_{f} R}\left[\frac{a_{c}}{R}\right]^{B} \int_{0}^{\tau} \xi \dot{P}_{i n c}(R, \xi) \mathrm{d} \xi\right\} \mathbf{e}_{\mathbf{R}}
\end{aligned}
$$

Finally, the incident pressure and velocity fields required for the fluid-structure interaction problem, respectively $\hat{p}_{\text {inc }}(1, \theta, s)$ and $\hat{\mathbf{v}}_{\text {inc }}(1, \theta, s) \cdot \mathbf{e}_{\mathbf{r}}$, are obtained by normalizing the relations $(37,38)$ and expressing them on the shell surface such as $p_{\text {inc }}(1, \theta, t)=P_{\text {inc }}(R(\theta), t)$ and $\mathbf{v}_{\text {inc }}(1, \theta, t)=\mathbf{V}_{\text {inc }}(R(\theta), t)$, with $R(\theta)$ the location of the shell external boundary,

$$
R(\theta)=\sqrt{(S+1)^{2}-2(S+1) \cos \theta+1}
$$

and $S$ the dimensionless stand-off. After a few manipulations, their analytical expressions in the Laplace domain take the following forms [39]:

$$
\begin{aligned}
\hat{p}_{\text {inc }}(1, \theta, s)= & P(\theta) C(\theta, s) \mathrm{e}^{-\beta(\theta) s} \\
\hat{\mathbf{v}}_{\text {inc }}(1, \theta, s) \cdot \mathbf{e}_{\mathbf{r}}= & \hat{p}_{\text {inc }}(1, \theta, s) \mathbf{e}_{\mathbf{R}} \cdot \mathbf{e}_{\mathbf{r}} \\
& +\left[V(\theta) D(\theta, s)+\frac{P(\theta) B}{R(\theta)} E(\theta, s)\right] \\
& \times \mathrm{e}^{-\beta(\theta) s} \mathbf{e}_{\mathbf{R}} \cdot \mathbf{e}_{\mathbf{r}}
\end{aligned}
$$

with the newly introduced quantities given by

$$
\begin{aligned}
P(\theta) & =\frac{P_{c}}{\rho_{f} c_{f}^{2}}\left[\frac{a_{c}}{R_{s} R(\theta)}\right]^{1+A} \\
V(\theta) & =\frac{P_{c} T_{c}}{\rho_{f} c_{f} R_{s}} \frac{1+A-B}{R(\theta)}\left[\frac{a_{c}}{R_{s} R(\theta)}\right]^{1+A-B} \\
C(\theta, s) & =\frac{0.8251}{s+1.338 \alpha(\theta)}+\frac{0.1749}{s+0.1805 \alpha(\theta)} \\
D(\theta, s) & =\frac{1.5856}{s}-\frac{0.6167}{s+1.338 \alpha(\theta)}-\frac{0.9690}{s+0.1805 \alpha(\theta)} \\
E(\theta, s) & =\frac{0.8251}{[s+1.338 \alpha(\theta)]^{2}}+\frac{0.1749}{[s+0.1805 \alpha(\theta)]^{2}} \\
\alpha(\theta) & =\frac{R_{s}}{T_{c} c_{f}}\left[\frac{a_{c}}{R_{s} R(\theta)}\right]^{B}, \beta(\theta)=R(\theta)-S
\end{aligned}
$$

The quantities $\hat{p}_{i n c}^{n}(s)$ and $\hat{v}_{i n c}^{n}(s)$ required in the computation of the column vector $\mathbf{f}_{\mathbf{n}}(s)$, equation (34), are directly obtained from numerical Fourier transforms of the relations (40).

\section{References}

[1] R.H. Cole, Underwater Explosions, Princeton University Press, 1948

[2] A.H. Keil, The response of ships to underwater explosions, Trans. Soc. Nav. Arch. Mar. Eng. 69 (1961) 33-41

[3] C.C. Liang, C.Y. Hsu, W.H. Lai, A study of transient response of a submerged spherical shell under shock waves, Ocean Eng. 28 (2000) 71-94

[4] Y. Shin, Ship shock modelling and simulation of far-field underwater explosion, Comp. Struct. 82 (2004) 22112219

[5] I.K. Park, J.C. Kim, C.W. An, D.S. Cho, Measurement of naval ship response to underwater explosion shock loading, Shock Vib. 10 (2003) 365-377

[6] F. Besnier, Simulation numérique et conception des structures de grands navires, Mécanique \& Industries 7 (2006) 213-222

[7] Y.S. Shin, L.D. Santiago, Surface ship shock modeling and simulation, Two-dimensional analysis, Shock Vib. 5 (1998) 129-137

[8] M.A. Sprague, T.L. Geers, A spectral element/finite element analysis of a ship like structure subjected to an underwater explosion, Comp. Meth. Appl. Mech. Eng. 195 (2006) 2149-2167

[9] Y. Le Bras, D. Vienne, Underwater explosion response of an ssk, In UDT Conference, Amsterdam, 2005

[10] C.C. Liang, Y.S. Tai, Shock response of surface ship subjected to noncontact underwater explosions, Ocean Eng. 33 (2006) 748-772

[11] Y.W. Kwon, R.E. Cunningham, Comparison of usa-dyna finite element models for a stiffened shell subjected to underwater shock, Comp. Struct. 66 (1998) 127-144

[12] H.U. Mair, Review: Hydrocodes for structural response to underwater explosion, Shock Vib. 6 (1999) 81-96 
[13] H.U. Mair, Benchmarks for submerged structure response to underwater explosion, Shock Vib. 6 (1999) 169-181

[14] J.H. Haywood, Response of an elastic cylindrical shell to a pressure pulse, J. Mech. Appl. Math. 11 (1958) 129-141

[15] S. Iakovlev, External shock loading on a submerged fluidfilled cylindrical shell, J. Fluids Struct. 22 (2006) 9971028

[16] S. Iakovlev, Submerged fluid-filled cylindrical shell subjected to a shock wave: Fluid-structure interaction effects, J. Fluids Struct. 23 (2007) 117-142

[17] Y.W. Kwon, P.K. Fox, Underwater shock response of a cylinder subjected to side-on explosion, Comp. Struct. 48 (1993) 637-646

[18] S.C. Tang, D.H.Y. Yen, Interaction of a plane acoustic wave with an elastic spherical shell, J. Acoust. Soc. Am. 47 (1970) 1325-1233

[19] T.L. Geers, Residual potential and approximation methods for three-dimensional fluid-structure interaction problems, J. Acoust. Soc. Am. 49 (1971) 1505-1510

[20] H. Huang, Y.F. Wang, Asymptotic fluid-structure interaction theories for acoustic radiation prediction, J. Acoust. Soc. Am. 77 (1985) 1389-1394

[21] T.L. Geers, Doubly asymptotic approximations for transient motion of submerged structures, J. Acoust. Soc. Am. 64 (1978) 1500-1508

[22] T.L. Geers, C.A. Felippa, Doubly asymptotic approximations for transient motion of submerged structures, J. Acoust. Soc. Am. 73 (1983) 1152-1159

[23] A.G. Pathak, P.R. Stepanishen, Acoustic harmonic radiation from fluid-loaded infinite cylindrical elastic shells using elasticity theory J. Acoust. Soc. Am. 99 (1994) 573582

[24] A. Baillard, J.-M. Conoir, D. Décultot, G. Maze, A. Klauson, J. Metsaveer, Acoustic scattering from fluidloaded stiffened cylindrical shell: Analysis using elasticity theory, J. Acoust. Soc. Am. 107 (2000) 3208-3216

[25] T.L. Geers, Excitation of an elastic cylindrical shell by a transient acoustic wave, J. Appl. Mech. 36 (1969) 459-469

[26] T.L. Geers, Scattering of a transient acoustic wave by an elastic cylindrical shell, J. Acous. Soc. Am. 51 (1972) $1640-1651$
[27] H. Huang, An exact analysis of the transient interaction of acoustic plane waves with a cylindrical elastic shell, J. Appl. Mech. 37 (1970) 1091-1099

[28] S. Iakovlev, Interaction between a submerged evacuated cylindrical shell and a shock wave - Part I: Diffractionradiation analysis, J. Fluids Struct. 24 (2008) 1077-1097

[29] S. Iakovlev, Interaction between a submerged evacuated cylindrical shell and a shock wave - Part II: Numerical aspects of the solution, J. Fluids Struct. 24 (2008) 10981119

[30] S. Iakovlev, Interaction of a spherical shock wave and a submerged fluid-filled circular cylindrical shell, J. Sound Vib. 255 (2002) 615-633

[31] W.G. Neubauer, L.R. Drageonette, Observation of waves radiated from circular cylinders caused by an incident pulse, J. Acoust. Soc. Am. 48 (1970) 1135-1149

[32] A.C. Ahyi, P. Pernod, O. Gatti, V. Latard, A. Merlen, H. Uberall, Experimental demonstration of the pseudoRayleigh wave, J. Acoust. Soc. Am. 104 (1998) 2727-2732

[33] L. Derbesse, P. Pernod, V. Latard, A. Merlen, D. Décultot, N. Touraine, G. Maze, Acoustic scattering from complex elastic shells: visualization of $S_{0}, A_{0}$ and $A$ waves, Ultrasonics 38 (2000) 860-863

[34] M. Abramowitz, I.A. Stegun, Handbook of Mathematical Functions, Dover, 1970

[35] L. Brancik, Utilization of matlab in simulation of linear hybrid circuits, Radioeng. 12 (2003) 6-11

[36] X.L. Bao, P.K. Raju, H. Uberall, Circumferential waves on an immersed, fluid-filled elastic cylindrical shell, J. Acoust. Soc. Am. 105 (1999) 2704-2709

[37] R.S. Price, Similitude equations for explosives fired underwater, Technical Report R 80-299, NSWC, 1979

[38] K.S. Hunter, T.L. Geers, Pressure and velocity fields produced by an underwater explosion, J. Acoust. Soc. Am. 115 (2004) 1483-1496

[39] C. Leblond, Modélisation de phénomènes fortement instationnaires en milieux couplés, Application au dimensionnement de structures immergées aux explosions sous-marines, Ph.D. Thesis, Université de Nantes, France, 2007 